\title{
Teaching Image Processing with Hands on
}

Suresh K. V. ${ }^{1}$

${ }^{1}$ Department of Electronics \& Communication Engineering, Siddaganga Institute of Technology, Tumkur,

Karnataka, India

${ }^{1}$ sureshkvsit@sit.ac.in

\begin{abstract}
The paper presents personal experience of author in teaching the course "Image Processing" for UG students. It gives a systematic approach followed in teaching which yielded an exponential rise in enrolment for the subject. Further, it also justifies the increase in projects carried out by students in the area of Image processing. Hands on sessions helped in meeting the course outcomes and mapping the same with the programme outcomes. Concepts were well understood through project based learning
\end{abstract}

Keywords: Image processing, hands on, project based learning, Programme outcomes (PO), Course outcomes (CO)

\section{Suresh K. V.}

${ }^{1}$ Department of Electronics \& Communication Engineering, Siddaganga Institute of Technology, Tumkur,

Karnataka, India

${ }^{1}$ sureshkvsit@sit.ac.in

\section{Introduction}

The saying " One picture is worth more than ten thousand words (Gonzalez and Woods 2012) no longer is true now. With the increase in the quality of images, "A picture is now worth more than a lakh words". When it comes to teaching a course on Image Processing, concepts penetrate only if appreciated. The appreciation from the student is expected only with Hands on. The author had really tough time in driving the concepts. Mere power point presentations along with the results from (Gonzalez and Woods 2012) during the initial stages of teaching did not help. The subject being an elective was not preferred by the students. This experience had led the author to research on innovative methods of teaching the course. Faculty teaching this course usually use problem based learning (Ambika 2015, Kolmos, 2012) for better delivery. Attempts were also made to improve the assessment by integrating laboratory and mini projects (Bhagyalaksmi et. al, 2015.)

Motivated with such efforts, the author tried lab based teaching. To begin with, a Graphical user interface (GUI) was developed using MATLAB image processing tool box (Gonzalez and Woods 2012). The concepts were explained along with demos using the GUI. This kindled the interest of the students and there was improvement in participation of students in the class room discussion. Fig. 1 gives the front end of the GUI created for the demo. The "Next" button allows the user to move to the next window which helps the users to perform simple operations on images. Flexibility is given to the users to select any image of their choice and change parameters to observe the changes. In the next sections, the details of the demos are provided.

\section{GUI for Image Processing}

This section provides the various operations that can be performed using the GUI. On pressing the "Next" button (Fig. 1), a new window (Fig. 2) opens. 


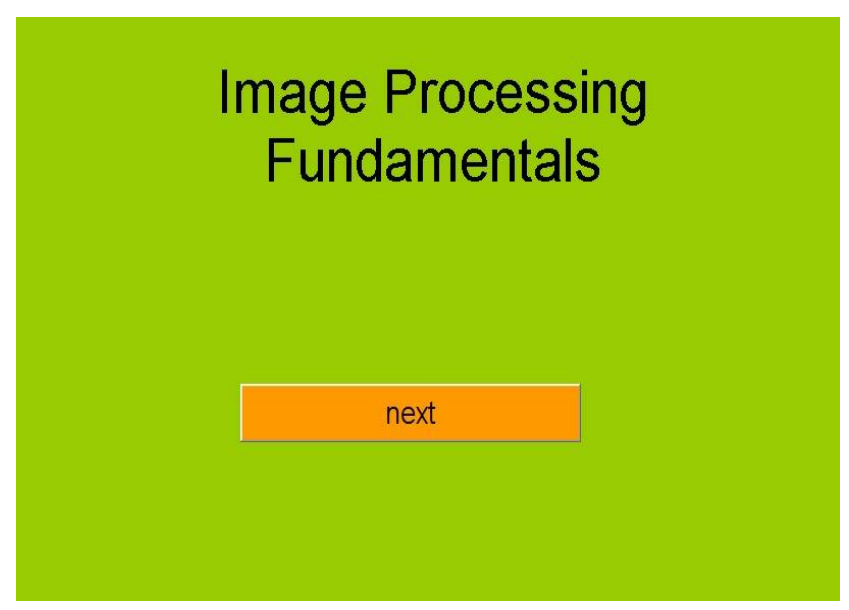

Fig.1 : Front end of GUI demo.

It can be observed from Fig. 2 that, the concepts such as effect of varying intensity resolution, spatial resolution can easily be observed. For example, we see false contouring effect when the brightness resolution is varied. Fig. 3 demonstrates this effect for a resolution of 4 bits. The user can further modify the brightness levels and observe the changes in the image.

More examples on few more concepts of Image processing are given in Fig. 4 and Fig. 5.

\section{Mini Projects on Image Processing}

Demonstrations of various concepts of Image Processing techniques using the GUI on MATLAB inspired the students to do hands on. Project based teaching was experimented. The students were asked to form

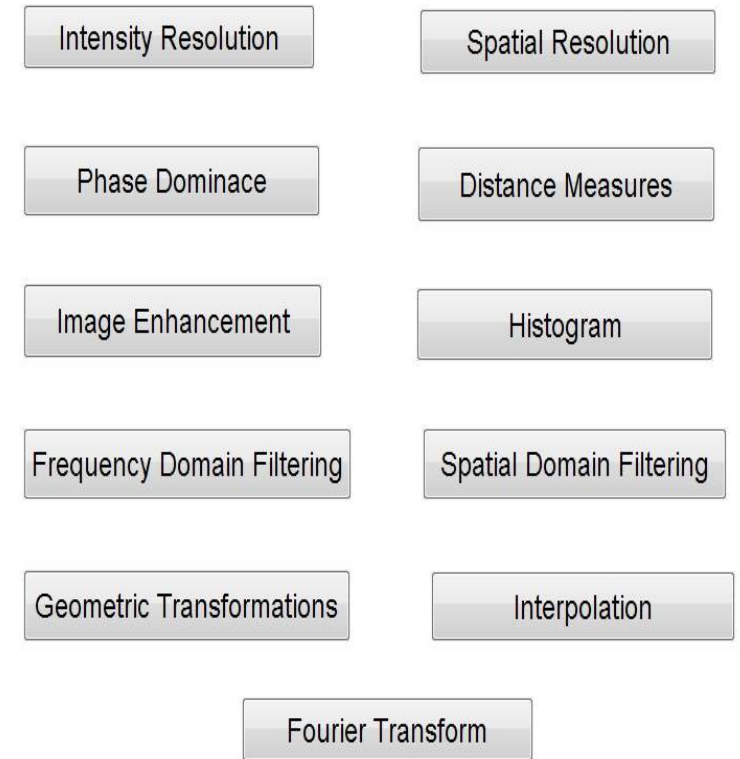

Fig. 2 : New window of GUI.

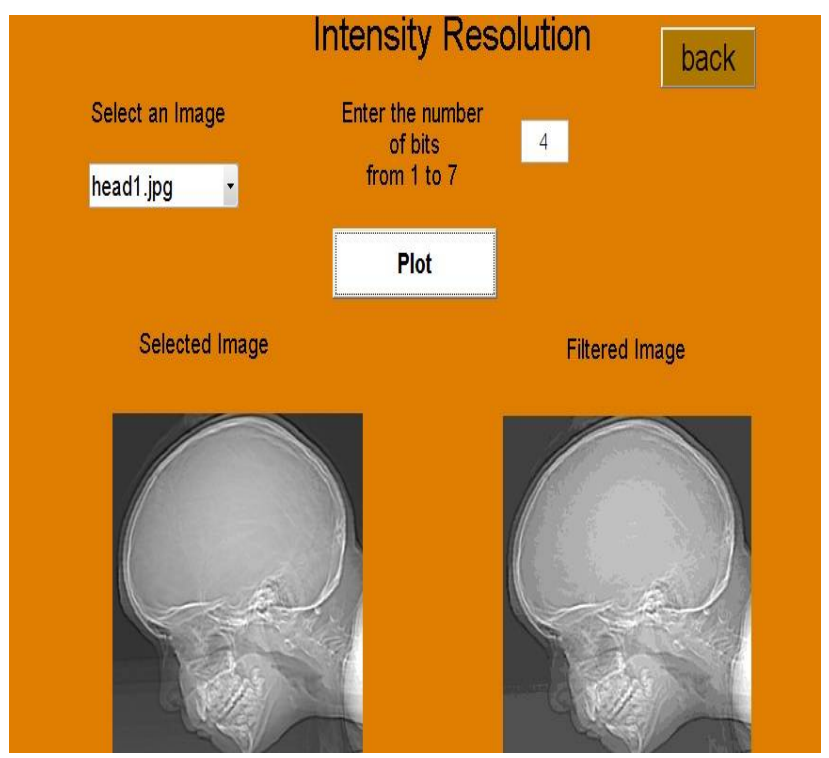

Fig. 3 : Demonstration of false contouring.

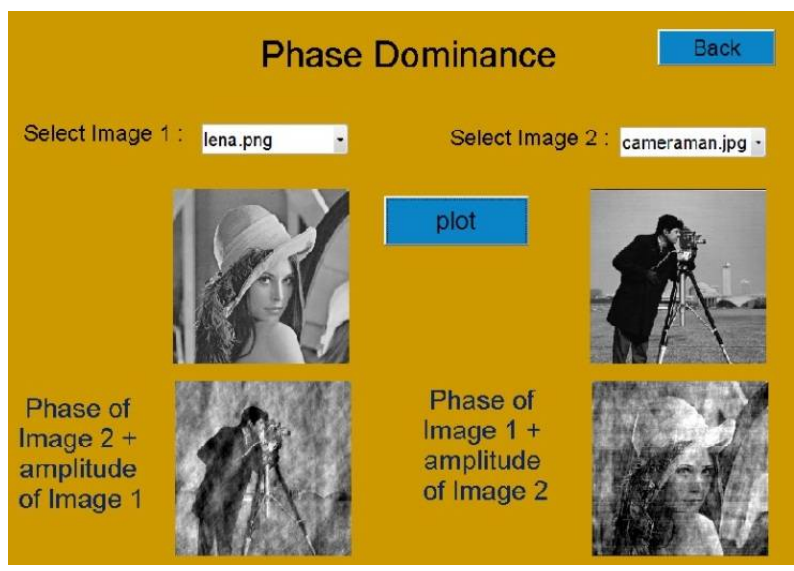

Fig. 4 : Demonstration of phase dominance in an image.

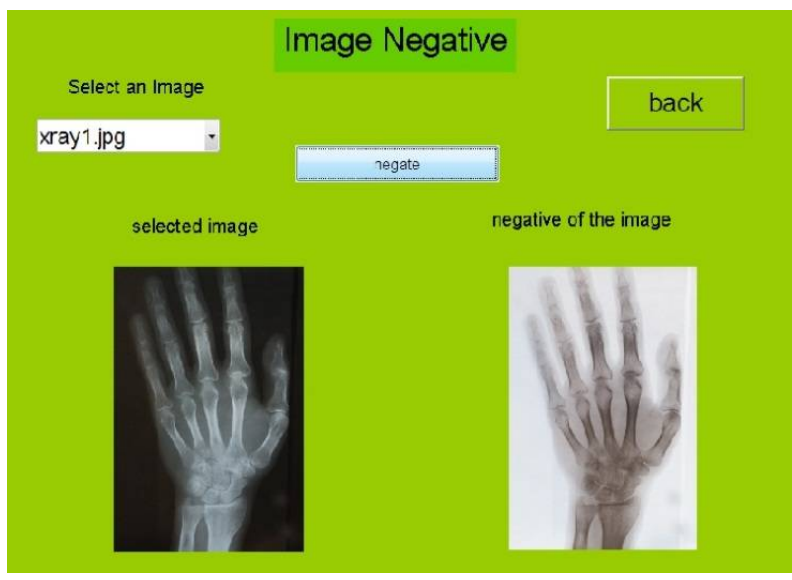

Fig. 5 : Demonstration of finding negative of an image. 
subgroups among themselves to carry out miniprojects on MATLAB. To motivate the students, out of 50 marks of continuous Internal Evaluation (CIE), 15\% weightage was given for successful completion of the miniproject. Students enthusiastically participated in developing the algorithms. Few ideas were suggested in the class and the rest came from the students themselves. The list of miniprojects carried out were

1. Calculation of area of an object with arbitrary shape

2. Measurement of distance between two points of an image from Google earth

3. Object Counting

4. Digital water marking

5. Image encryption and decryption

6. Face detection

7. Correlation based Fingerprint matching

8. GUI for image enhancement in spatial domain

9. GUI for image enhancement in frequency domain

10. Homomorphic filtering

11. Licence plate detection

12. Labelling of connected components

13. Pseudocolor processing

14. Resistor identification based on color code

15. ROI enhancement

16. Shape detection and counting

17. Intruder detection

All the projects were demonstrated by the respective group to their peers. A brief report comprising the basics, methodology used, and the algorithm was submitted in the end. This exercise not only helped the students in applying the basics learnt in the class to develop the algorithm but also gave ample opportunity to work in a team. Further, their oral and written communication was improved.

As a case study, we now present one of the miniprojects developed for measurement of distance between two points of an image from Google earth. Euclidean distance is a measure to determine the distance between two points $p(x, y)$ and $q(s, t)$ in an image given by

$$
D_{e}(p, q)=\sqrt{(x-s)^{2}+(y-t)^{2}}
$$

This concept can be used to determine the distance between any two places in a Google earth image. A batch of students worked on this idea. Fig. 6 (a) gives the front end of the GUI giving option to user to choose the Google earth image. Upon choosing the image, calibration with respect to the actual distance is performed. Now, by choosing the two points on the image, distance between two places can be determined. Fig. 6 (b) gives the result. The Google earth image selected by the students was interestingly the area showing "SIT, Tumkur". The distance between main building and the main road was measured to be equal to 361.3 meters as depicted in Fig. 6 (b).

Fig. 7 gives another example of a miniproject developed to determine the shape and count the same.

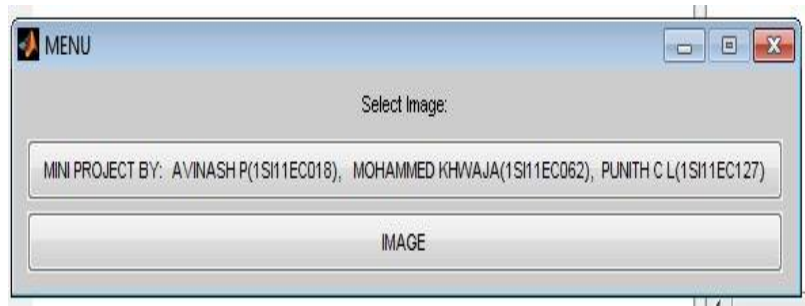

(a) GUI for finding distance

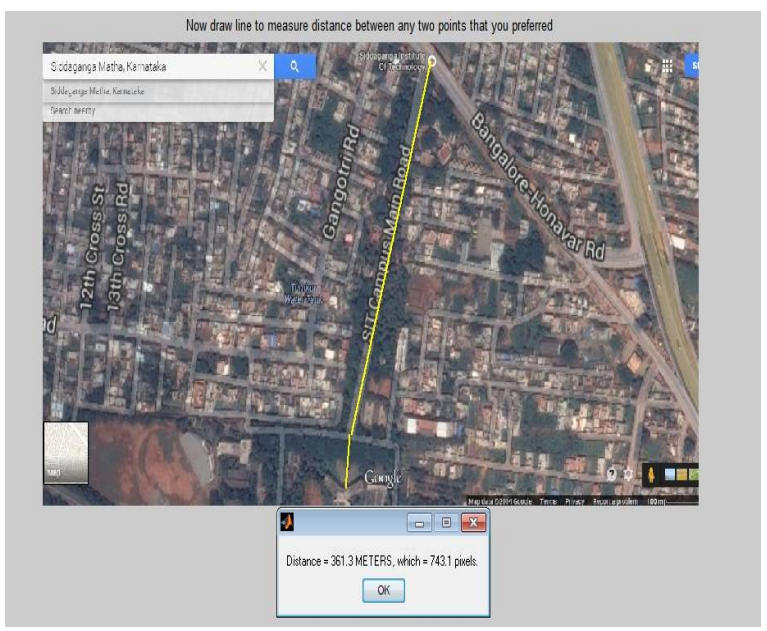

(b) An example showing the distance.

Fig. 6 : An example of distance measurement. 


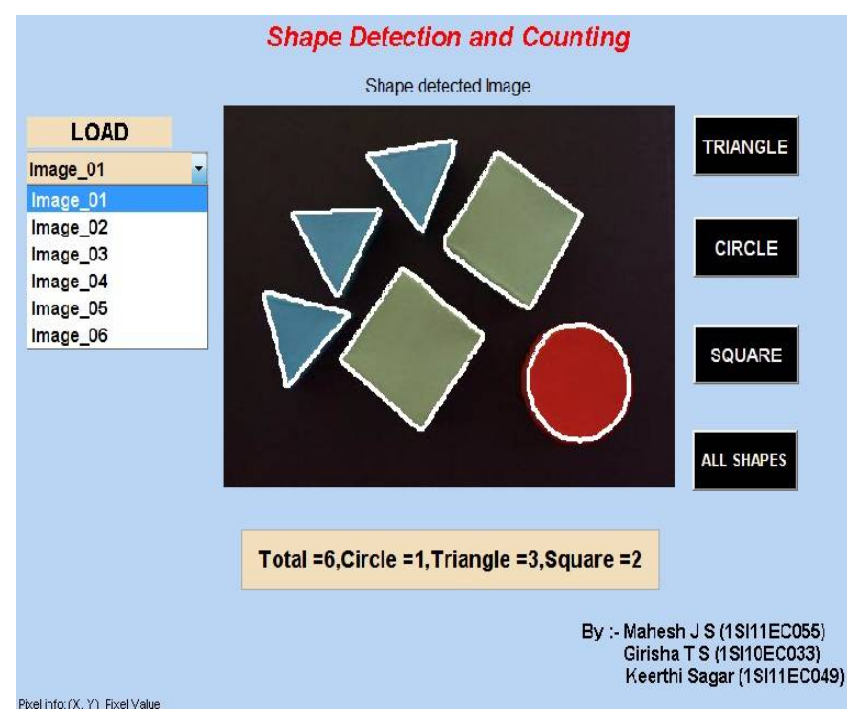

Fig. 7 : An example of shape detection and counting.
The enthusiasm kindled among students through the miniprojects motivated them to select projects based on image processing. About $25 \%$ of the major projects carried out in the academic year were on Image Processing. The list of a few projects are

1. People counting based on image processing

2. E-ration management based on face recognition

3. Currency recognition using image processing

4. Driver Drowsyness detection

As a case study, project titled "People counting based on image processing" (Gokul et. al. 2014) is presented here. Counting people in big seminar halls, auditoriums, shopping Mals is of at most importance for management. An attempt is made in this project to count people and display the number of occupants at any given time in various places. Head movement and direction is determined based on image processing. Fig. 8 gives the block diagram of the counting system and Fig. 9 displays the camera arrangement for the same. The count of students entering PG class room is given in Fig. 10. The project was adjudged as "Best project of the year" and the outcome of the project was also presented in a national conference which was given the "Best paper award in DSP domain". A local firm has also come forward to develop the idea into a product.

\section{Major Projects}

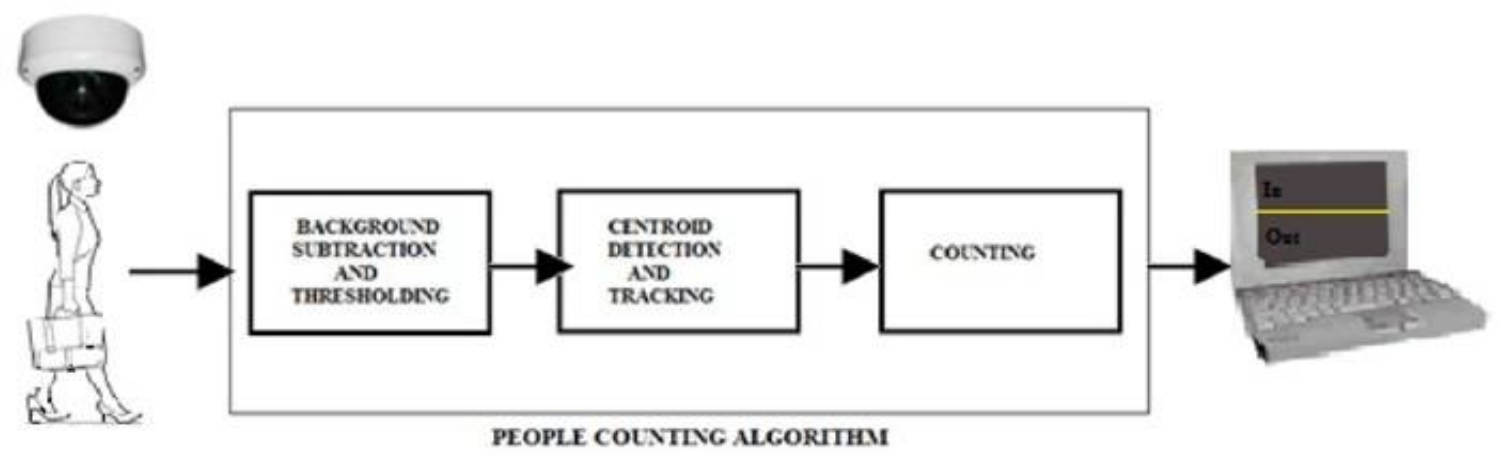

Fig. 8 : Block diagram of people counting system. 


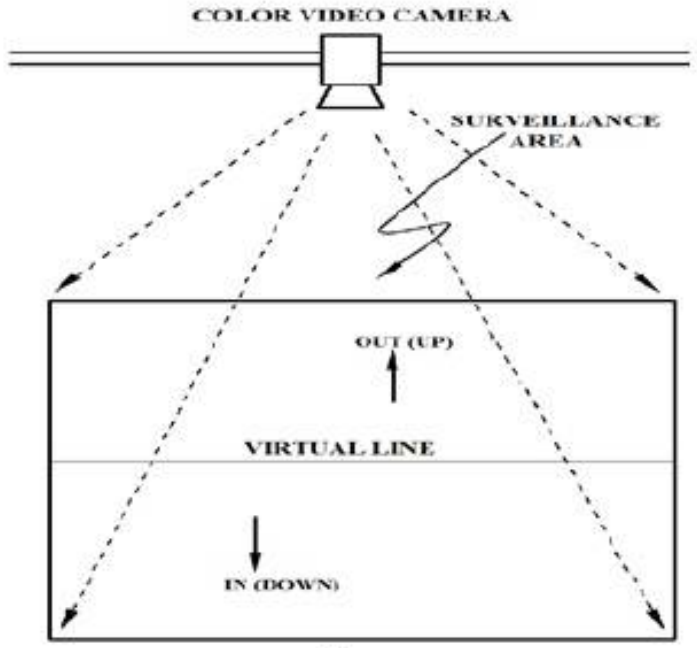

(a)

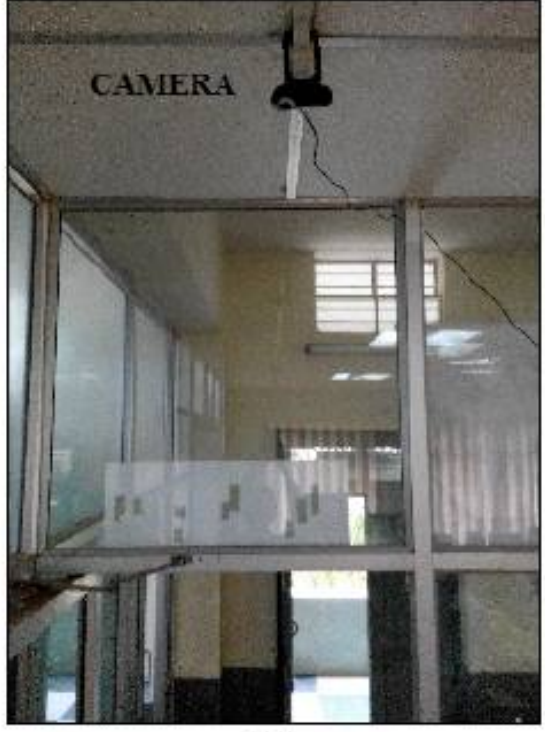

(b)

Fig. 9 : Camera arrangement

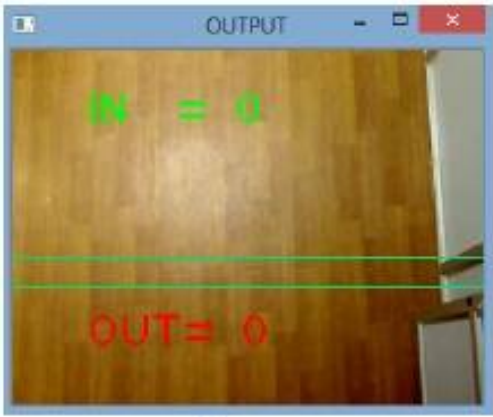

(a)

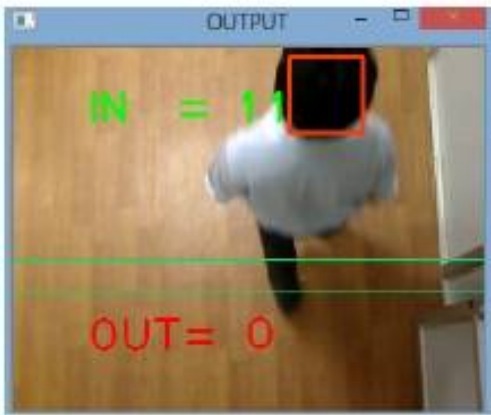

(b)

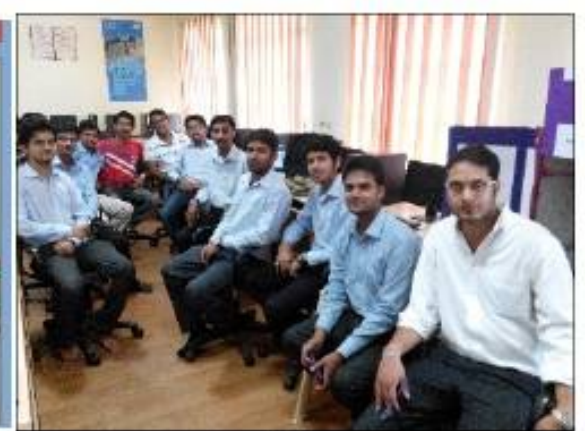

(c)

Fig. 10 : Demonstration in PG class room.

\section{CO-PO Mapping}

The course "Image Processing" is a professional elective with the following course outcomes $(\mathrm{CO})$.

A student who successfully completes this course should be able to:

1. define various terminologies used in Digital Image processing (L1)

2. identify and explain various steps and components used in digital image processing (L2)

3. analyze Images in transform (L3)
4. apply image processing techniques in both spatial and frequency domains (L3)

5. write simple image processing algorithms using software tools (L2)

6. experience working in teams ( L3)

7. communicate effectively on latest trends in Image processing (L3)

Knowledge levels :

\section{L1:Remembering, L2: Understanding,}

\section{L3: Applying, L4:Analyzing,}




\section{L5:Evaluating, L6:Creating}

The subject addresses the following programme outcomes (PO).

At the end of the programme, graduate of Electronics and communication engineering programme will be able to

PO: a apply knowledge of mathematics, sciences, and engineering to solve engineering problems in the area of electronics and communication

PO: b identify, formulate and analyze problems in the area of microelectronics, communication and embedded systems

PO: c design/implement electronic subsystem, signal processing algorithm and an embedded system to meet desired needs considering environmental, social, ethical, economical, health and safety issues

PO: $\mathbf{d}$ conduct investigations of complex problems that cannot be solved by straight forward application of knowledge and that which may not have unique solution considering appropriate constraints which may not have been specified in the problem

PO: e use modern engineering tools/software to analyze and design systems

PO: $\mathbf{h}$ demonstrate knowledge of professional and ethical responsibilities

PO: i work effectively as an individual and as a member or leader in diverse teams and in multidisciplinary settings

PO: $\mathbf{j} \quad$ communicate effectively both in oral and written form

PO: 1 develop confidence for self education and life long learning

\section{CO-PO Mapping}

\begin{tabular}{|l|l|l|l|l|l|l|l|l|l|l|l|l|}
\hline $\begin{array}{l}\text { PO } \\
\text { CO }\end{array}$ & a & b & c & d & e & f & g & h & i & j & k & l \\
\hline $\mathbf{1}$ & $\mathrm{X}$ & & & & & & & & & & & \\
\hline $\mathbf{2}$ & $\mathrm{X}$ & & & & & & & & & & & \\
\hline $\mathbf{3}$ & $\mathrm{X}$ & $\mathrm{X}$ & & & & & & & & & & \\
\hline $\mathbf{4}$ & $\mathrm{X}$ & $\mathrm{X}$ & & $\mathrm{X}$ & & & & & & & & \\
\hline $\mathbf{5}$ & $\mathrm{X}$ & $\mathrm{X}$ & $\mathrm{X}$ & $\mathrm{X}$ & $\mathrm{X}$ & & & & & & & \\
\hline $\mathbf{6}$ & & & & & & & & & $\mathrm{X}$ & & & \\
\hline 7 & $\mathrm{X}$ & & & & & & & $\mathrm{X}$ & & $\mathrm{X}$ & & $\mathbf{X}$ \\
\hline
\end{tabular}

(PO:GA : a:Engineering Knowledge, b:Problem analysis, c: design/development of solutions, d:conduct investigations of complex problems, e: modern tool usage, f:engineer and society, g:environment and sustainability, h:ehics, i:individual and team work, j:communication, k:project management and finance, l:life long learning)

\section{Conclusions}

The paper presents an attempt made to improve the teaching method specific to the course "Image Processing". The technique employed helped to address various graduate attributes specified by ABET. Hands on with MATLAB helped the students to appreciate the subject. With the new method of course delivery and evaluation, the subject could address many graduate attributes.

\section{References}

R.C. Gonzalez and R. E. Wooods (2012), Digital Image Processing, $3^{\text {rd }}$ Edition, Pearson Education, Asia

Ambika D. R. (2015) Project based learning in Digital Image Processing courser, Proc. $2^{\text {nd }}$ International
Conference on Transformations in Engineering Education, . 87-92 
Annette Kolmos (2012), Changing the curriculum to problem based and project based learning.

Bhagyalakshmi Lalitha and Harishanand (2015) "Continuous improvement in the assessment of image processing course, Proc. $2^{\text {nd }}$ International Conference on Transformations in Engineering Education, 885-887.

R.C.Gonzalez and R.E. Woods (2012), Digital image processing with matlab, Pearson, Education Asia

Gokul K B., A. Chandan, Avinash P and E. Y .Eashwara Murthy (2014), Real time people counting using image processing, Project carried out at SIT, Tumkur submitted to VTU, Belgaum. 